\title{
The Utility of Endoscopic Ultrasound-Guided Brachytherapy in Liver Metastasis: A Case Report and Review of the Literature
}

\author{
Nayana E. George ${ }^{1}$, Benjamin Tharian ${ }^{1}$, Helen Lyo ${ }^{1}$, Apoorva Jayarangaiah ${ }^{2}$, Irini Youssef ${ }^{1}$, \\ Joie Singh ${ }^{1}$, Samy I. McFarlane ${ }^{1, *}$, Shivakumar Vignesh ${ }^{3}$ \\ ${ }^{1}$ Department of Medicine, State University of New York-Downstate Medical Center, Brooklyn, NY 11203 \\ ${ }^{2}$ Department of Internal Medicine, Wake Forest University, Baptist Health Care System, Winston-Salem, \\ North Carolina, U.S.A- 27157 \\ ${ }^{3}$ Division of Gastroenterology, Department of Medicine, State University of New York-Downstate Medical Center, \\ Brooklyn, NY 11203 \\ *Corresponding author: Samy.mcfarlane@downstate.edu
}

Received August 07, 2018; Revised October 01, 2018; Accepted October 14, 2018

\begin{abstract}
Endoscopic ultrasound guided brachytherapy (EGBT) has been reported to be useful in certain malignancies including esophageal and pancreatic cancers. Percutaneous or surgical placement of radioactive seeds into the liver secondaries (brachytherapy) has been done successfully, however, the utility of EGBT in liver metastasis remains largely unclear. In this case report, we demonstrate the safety, efficacy and feasibility of EUS guided brachytherapy (EGBT) in liver metastasis secondary to leiomyosarcoma.
\end{abstract}

Keywords: endoscopic ultrasound guided brachytherapy, liver metastasis

Cite This Article: Nayana E. George, Benjamin Tharian, Helen Lyo, Apoorva Jayarangaiah, Irini Youssef, Joie Singh, Samy I. McFarlane, and Shivakumar Vignesh, "The Utility of Endoscopic Ultrasound-Guided Brachytherapy in Liver Metastasis: A Case Report and Review of the Literature." American Journal of Medical Case Reports, vol. 6, no. 9 (2018): 189-192. doi: 10.12691/ajmcr-6-9-5.

\section{Introduction}

Metastatic leiomyosarcoma (LMS) typically carries a poor prognosis.

Liver metastases are managed with multimodal therapies including segmental hepatectomy, systemic chemotherapy or local ablative therapies. Image guided therapies include targeted chemotherapy, ethanol ablation, radiofrequency laser, cryotherapy, transarterial chemoembolization (TACE) and interstitial brachytherapy with radioactive seeds. Regional therapies minimize systemic toxicity without compromising the tumoricidal effects.

Linear echo-endoscopes enable fine needle injection (FNI) under real time image guidance. Its therapeutic applications in oncology include, but are not limited to, injection of cytotoxins, celiac plexus neurolysis (CPN), radiofrequency ablation (RFA), radiation therapy (fiducial placement and brachytherapy), biliary access and palliative anastomoses. Our patient had EGBT for liver metastases, with deployment of Iodine-125 (I-125) seeds. This is the first report of EGBT for liver metastasis.

\section{Case Report}

We report a 73-year-old lady, who underwent EGBT for retroperitoneal soft tissue sarcoma (STS) with liver metastases. The technical aspects of the procedure and follow up are described in this article.

The patient presented in 2007, with a $12.6 \mathrm{~cm}$ pelvic mass secondary to a high-grade LMS arising from the sigmoid mesentery which was excised surgically. She had no past history except for vaginal hysterectomy for cervical dysplasia. She opted for a "wait and watch" approach. Surveillance imaging in 2009 revealed a liver lesion $((13 \mathrm{~mm}$ in segment 8 (a)). She had Adriamycin Ifosfamide chemotherapy and subsequent percutaneous hepatic perfusion (CS-PHP) of Melphalan. Her Eastern Cooperative Oncology Group status (ECOG) score was 0 and Karnofsky Performance Scale was 100 on completion of 4 cycles in July 2010. Subsequent imaging in June 2011 revealed progression of liver metastases and Virchow's node requiring radical neck dissection and metastasectomy. New metastases appeared in segment $2(22 \times 24 \mathrm{~mm}$ ' $\mathrm{b}$ ', and $8 \mathrm{~mm}$ lesion 'c') and segment 4 (27x26 mm 'd'), with interval progression of ' $a$ ' in January 2012.

The patient underwent EGBT into the left lobe lesion (Figure 1) along with radioembolization of the right lobe with Yttrium (Y-90), transarterial radioembolization (TARE) and stereotactic external beam radiotherapy (SBRT) for the central lesions. EGBT was done by the advanced endoscopist (SV) under monitored anaesthesia care (Figure 2, Figure 3, Figure 4). Four I-125 seeds were preloaded per strand. Linear echoendoscope GF-UC 140P 
was used to identify target lesions within the left lobe. The tip of the 19-G Cook needle was loaded with the seeds and sealed using bone wax. Under EUS guidance, the needle was advanced to the distal margin of the lesion and the strands deployed, simultaneously withdrawing the needle and advancing the stylet. Sixteen seeds were placed in all. Interval imaging revealed a different lesion measuring $26 \times 25 \mathrm{~mm}$ in the left lobe, requiring further EGBT nine weeks later, deploying a total of nineteen seeds. The procedure was well tolerated with no complications.

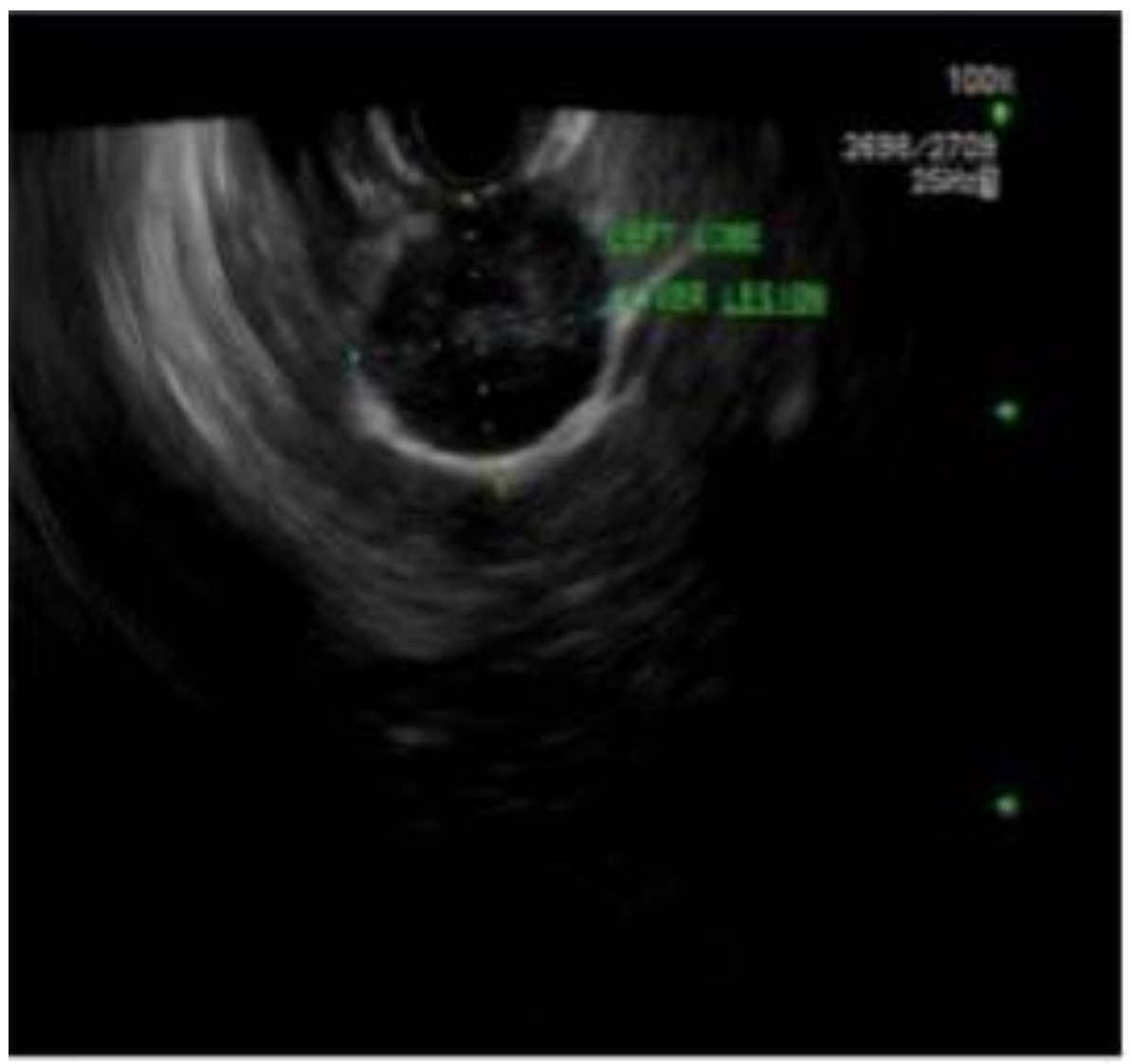

Figure 1. EUS showing the left liver lobe lesion

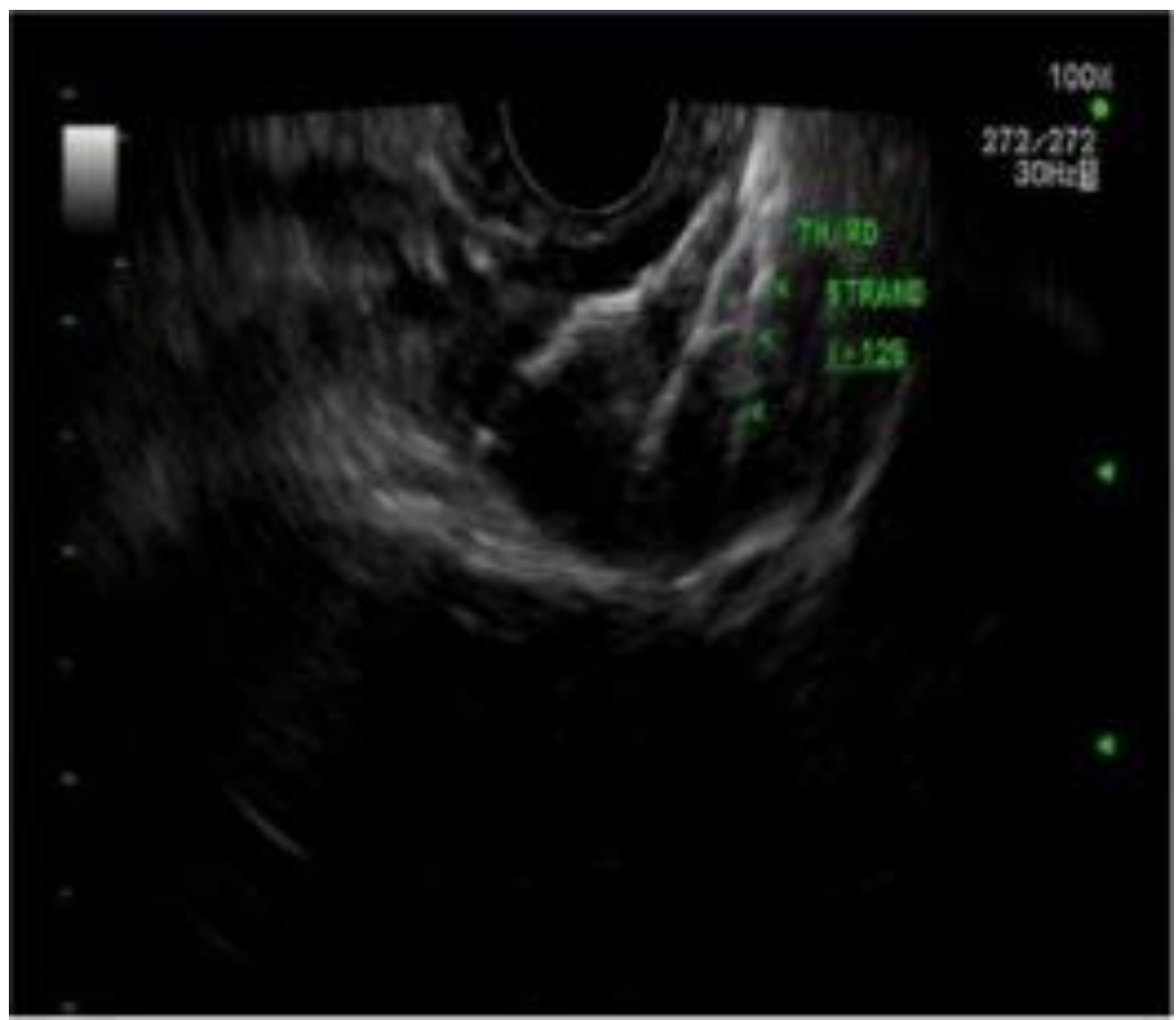

Figure 2. EUS showing deployed strands 


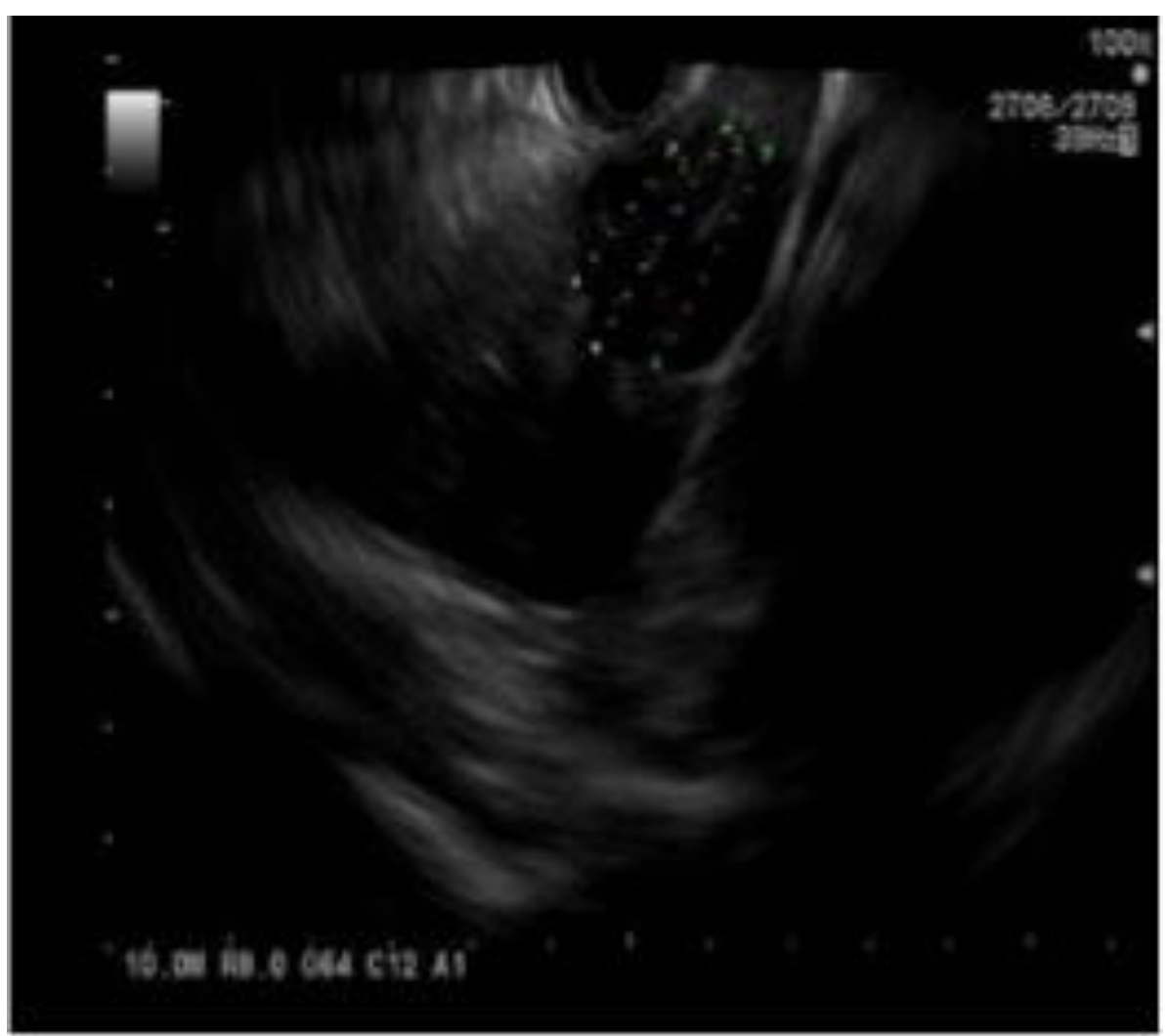

Figure 3. EUS showing I-125 Seeds within the lesion

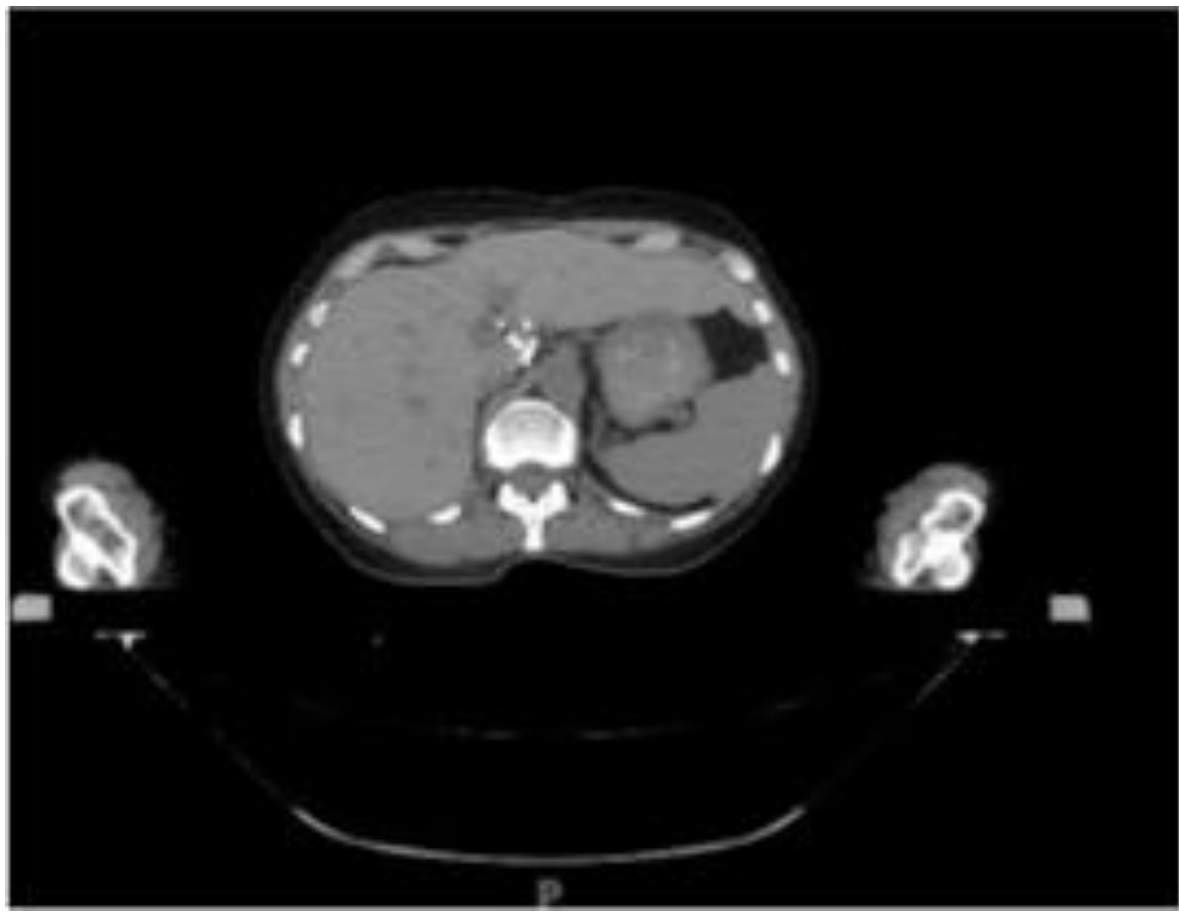

Figure 4. CT showing the seeds

Positron emission tomography (PET) demonstrated minimal increase in metabolic activity within the liver lesions. CT guided biopsy of FDG avid liver lesions done nine months after initial EGBT, to exclude post-inflammatory changes, confirmed that the increased metabolic activity was secondary to metastasis. She received further TARE and SBRT, but developed therapy related Myelodysplastic Syndrome. She was palliated and died in July 2013, four years after the initial appearance of liver metastases.

\section{Discussion}

Liver metastasis most commonly arise from colon cancer, melanoma, neuroendocrine tumors and other 
visceral malignancies, including STS. It portends a poor prognosis with a median survival of one year [1]. Resection offers the highest survival advantage for isolated liver metastasis [2]. Only $17 \%$ were eligible for resection, with a 3 year survival of $50 \%$ in a large series from Memorial Sloan-Kettering Cancer Center [2]. Vast majority are palliated, with the exceptional patient being a transplant candidate. Palliation involves a combination of systemic chemotherapy, SBRT, radiotherapy or locally ablative treatments.

Linear EUS is evolving in endoscopic Oncology. It is a minimally invasive intervention for pancreatico-biliary drainage, intratumoral injection of agents and fiducial marker placement to facilitate image guided radiation therapy (IGRT) and SBRT [3]. An advantage of low dose rate brachytherapy is that it allows for a more concentrated effect on the targeted tumor [3]. Additionally, the short penetration distance minimizes the effects of radiation on the surrounding healthy tissue [3]. Radiotherapy is limited since radio tolerance of normal liver tissue is around $35 \mathrm{~Gy}$, much below the tumoricidal dose for hepatic malignancies, which is 70Gy [4]. Brachytherapy was introduced in the 50's and had been done by implanting seeds during laparotomy or by percutaneous approach [5].

EGBT is similar to fiducial placement, but uses radioactive seeds instead of an inert marker. Seeds used include I-125, Iridium-192 and Palladium-103. I-125 is preferred due to longer half life of 59.7 days and is ideal for rapidly growing tumors including metastases [6].

Initial trials of brachytherapy in humans were on head and neck tumors and recently on the pancreas [7,8]. The trials done by Sun et al are the only available human trials addressing this. They used a mean of 22 seeds per patient with $100 \%$ technical success and reported median survival of 10.6 months with $27 \%$ showing partial tumor response [9]. Guo et al used EGBT in retroperitoneal malignancy [10].

Management of patients with liver metastases, especially those from soft tissue sarcomas remain challenging. This report demonstrates a patient centered approach for an aggressive metastatic malignancy. Our patient maintained good performance status for over 6 years, for a condition with 3-year survival of $50 \%$. Shorter seeds, newer particles and liquid formulation would enable easier delivery. Better software programs for treatment planning based on EUS imaging would rationalize dose calculation and more precise deployment of seeds with improved tumoricidal effects.

\section{Conclusion}

Our anecdotal experience is the first report of EGBT of liver metastases with encouraging results and holds promise as a new treatment for metastatic cancer. The patient had a survival benefit, from a multimodal approach involving two investigational treatments; CS-PHP and EGBT, both have been safe with some success. EGBT is precise and less invasive and targets multiple lesions. We demonstrated its technical feasibility, safety and tolerability. Randomized multicenter trials are needed, before making firm recommendations for EGBT.

\section{Acknowledgements}

This work is supported, in part, by the efforts of Dr. Moro O. Salifu M.D., M.P.H., M.B.A., M.A.C.P., Professor and Chairman of Medicine through NIH Grant number S21MD01247.

\section{References}

[1] Jaques DP, Coit DG, Casper ES, Brennan MF. Hepatic metastases from soft-tissue sarcoma. Ann Surg. 1995 Apr; 221(4): 392-7.

[2] DeMatteo RP, Shah A, Fong Y, Jarnagin WR, Blumgart LH, Brennan MF. Results of hepatic resection for sarcoma metastatic to liver. Ann Surg. 2001 Oct; 234(4): 540-7; discussion 547-8.

[3] Jin Z, Chang KJ. Endoscopic ultrasound-guided fiducial markers and brachytherapy. Gastrointest Endosc Clin N Am. 2012 Apr; 22(2): 325-31, x.

[4] Seidensticker M, Wust P, Rühl R, et al. Safety margin in irradiation of colorectal liver metastases: assessment of the control dose of micrometastases. Radiat Oncol Lond Engl. 2010; 5: 24.

[5] Collettini F, Singh A, Schnapauff D, et al. Computed-tomographyguided high-dose-rate brachytherapy (CT-HDRBT) ablation of metastases adjacent to the liver hilum. Eur J Radiol. 2013 Oct; 82(10): e509-14.

[6] Erickson RA. "Hot stuff': EUS-guided brachytherapy. Gastrointest Endosc. 2005 Nov; 62(5): 808-10.

[7] Sun S, Xu H, Xin J, Liu J, Guo Q, Li S. Endoscopic ultrasoundguided interstitial brachytherapy of unresectable pancreatic cancer: results of a pilot trial. Endoscopy. 2006 Apr; 38(4): 399-403.

[8] Lah JJ, Kuo JV, Chang KJ, Nguyen PT. EUS-guided brachytherapy. Gastrointest Endosc. 2005 Nov; 62(5): 805-8.

[9] Sun S, Qingjie L, Qiyong G, Mengchun W, Bo Q, Hong X. EUSguided interstitial brachytherapy of the pancreas: a feasibility study. Gastrointest Endosc. 2005 Nov; 62(5): 775-9.

[10] Guo Y, Liu Y, Li Z, et al. EUS-guided implantation of radioactive iodine-125 seeds in retroperitoneal metastatic adenocarcinoma. Endoscopy. 2009; 41 Suppl 2: E301. 